\section{Subpollen-Partikeln von Ragweed auf der Spur}

\author{
Zwar korrelieren Pollenflugsaison und allergische Symptome eng, \\ doch sind es nicht direkt die Pollenkörner, sondern kleinere, aus den \\ Pollen freigesetzte Partikel, welche die allergische Reaktion auslösen. \\ In einer Experimentalstudie wurde der entzündungsinduzierende \\ Mechanismus genauer untersucht.
}

E ntscheidend für die Auslösung einer entzündlichen allergischen Atemwegsreaktionen ist nicht unbedingt eine hohe aktuelle Pollenkonzentration, sondern vielmehr eine hohe Konzentration aus den Pollen freigesetzter Partikel. Diese kleinen Partikel werden als „Subpollen-Partikel“ (SPP) bezeichnet und sind aufgrund ihrer geringen Größe gut lungengängig. SPP enthalten neben spezifischen Allergenen häufig noch weitere Substanzen, die das entzündliche Geschehen im Lungengewebe fördern können.

Die SPP von Ragweed-Pollen ( $\mathrm{Am}$ brosia artemisiifolia) wurden von einer Gruppe von Wissenschaftlern aus dem US-Bundesstaat Texas näher untersucht. In einem ersten Schritt wurden die Ragweed-Pollenkörner in Kontakt mit Wasser gebracht, dadurch wurde die Freisetzung der SPP in einer Größe zwischen 0,5 und $4,5 \mu \mathrm{m}$ induziert. Die Partikel enthielten das Ragweed-
Hauptallergen Amb a 1 sowie andere allergen wirkende Proteine. Daneben zeigten die SPP auch NAD(P)H-Oxidase-Aktivität. In Zellkulturen steigerten

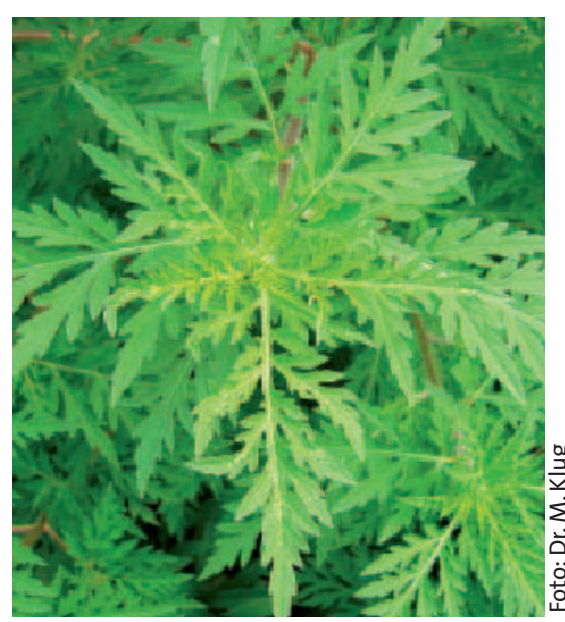
finden: das Traubenkraut, englisch Ragweed, lateinisch Ambrosia artemisiifolia
Auch in Europa immer häufiger zu die Partikel signifikant die Spiegel reaktiver Sauerstoffspezies (Sauerstoffradikale) und induzierten im Tierexperiment eine allergische Atemwegsentzündung.

Die Autoren kommen zu dem Schluss, dass der durch die NAD(P)HOxidase-Aktivität erzeugte oxidative Stress unmittelbar nach dem Kontakt mit den Atemwegszellen entsteht und offensichtlich die Allergen-induzierte Entzündung in den unteren Atemwegen triggert. SPP können auch dann massenweise in der Luft vorhanden sein, wenn die Pollenzahl selbst relativ niedrig ist. $\mathrm{NAD}(\mathrm{P}) \mathrm{H}-\mathrm{Oxidasen}$ schließlich findet man nicht nur in Ragweed-Pollen, sondern auch in Pollenextrakten anderer Kräuter sowie von Gräsern und Bäumen mit allergener Potenz. Möglicherweise könnte die durch die winzigen Partikel induzierte allergische Entzündung durch Antioxidanzien oder Oxidaseinhibitoren abgemildert werden.

Fazit: Subpollen-Partikel aus RagweedPollenkörnern werden nach Wasserkontakt freigesetzt und führen durch die "Tandemwirkung" von intrinsischen $\mathrm{NAD}(\mathrm{P}) \mathrm{H}-\mathrm{Oxidasen}$ und Allergenen zu einer allergischen Entzündung.

$b k$

Bacsi A et al. Subpollen particles: carriers of allergenic proteins and oxidases. J

Allergy Clin Immunol 2006; 118: 844-50

\title{
Näher an der Autobahn - weniger Luft für Kinder
}

\section{Welchen Effekt hat die Exposition gegenüber Autoabgasen auf die Entwicklung der Lungenfunktion? US-amerikanische Forscher gingen dieser Frage in einer prospektiven Studie nach, indem sie Kinder und Jugendliche über acht Jahre hinweg beobachteten.}

\footnotetext{
nsgesamt 3.677 Kinder aus verschiedenen Gemeinden in Südkalifornien nahmen an einer prospektiven Untersuchung teil. Die Kinder waren zu Studienbeginn alle im Schnitt zehn Jahre alt. Bis zu ihrem 18. Lebensjahr wurden bei den Kindern jedes Jahr Lungenfunktionsprüfungen durchgeführt.
}

Für die Exposition gegenüber Schadstoffen bildeten die Wissenschaftler - je nachdem, in welcher Entfernung die Kinder von einer viel befahrenen Straße (Autobahn) lebten - vier Hauptkategorien: weniger als $500 \mathrm{~m}$ Entfernung, 500-1.000 m, $1.001-1.500 \mathrm{~m}$ und mehr als $1.500 \mathrm{~m}$.
Im Laufe der acht Jahre nahmen bei den Studienteilnehmern die Lungenfunktionsparameter Vitalkapazität (VK), Einsekundenkapazität $\left(\mathrm{FEV}_{1}\right)$ und maximaler mittelexspiratorischer Fluss (MMEF) deutlich zu. Dabei war jedoch die Entwicklung der Lungenfunktion von der Entfernung des Wohnorts zur Autobahn abhängig. Wohnten die Kinder weniger als $500 \mathrm{~m}$ davon entfernt, nahmen $\mathrm{FEV}_{1}$ und MMEF in acht Jahren um $81 \mathrm{ml}$ bzw. um $127 \mathrm{ml}$ weniger zu, verglichen mit Studienteilnehmern, die weiter als 1.500 $\mathrm{m}$ entfernt lebten. Dieser Unterschied war signifikant. Auch in den Untergruppen der Nichtraucher und der Nichtasthmatiker stiegen hier die $\mathrm{FEV}_{1}$ - 